\title{
Comments on the Discrete Matrix Model of Population Dynamics
}

\author{
Ralph Freese,* and Charles R. Johnson \\ Institute for Basic Standards, National Bureau of Standards, Washington, D.C. 20234
}

(February 14, 1974)

\begin{abstract}
This paper examines several aspects of the discrete matrix model of population transition. Certain appropriate applications of matrix theory and exploitation of the specific form of the model should serve to enhance its already well-developed status. The aspects dealt with include (1) a simplification of the Perron-Frobenius theory; (2) row and column sum bounds on maximal eigenvalues; (3) relations between oscillations in a population and the remaining eigenvalues; (4) implications of stability for the transition matrix; and (5) relations between characteristic quantities of a stable population.
\end{abstract}

Key words: Eigenvalue; net reproduction rate; oscillations; Perron-Frobenius theory; rate of natural increase; stable population.

\section{Introduction}

The purpose of this paper is to examine certain aspects of the matrix model of population dynamics. We begin with a brief discussion of the model. More details may be found in Keyfitz (1968).

In the discrete model of population, the population at time $t$ is represented by a vector, $v(t)$. The $i$ th component of this vector, $v_{i}(t)$, is the number of people at time $t$ between the ages of $(i-1) \Delta t$ and $i \Delta t$ where $\Delta t$ is a fixed time period, usually one or five years.

The following matrix $A=\left(a_{i j}\right)$ acts on $v(t)$ to give $v(t+1)$, see Keyfitz (1968):

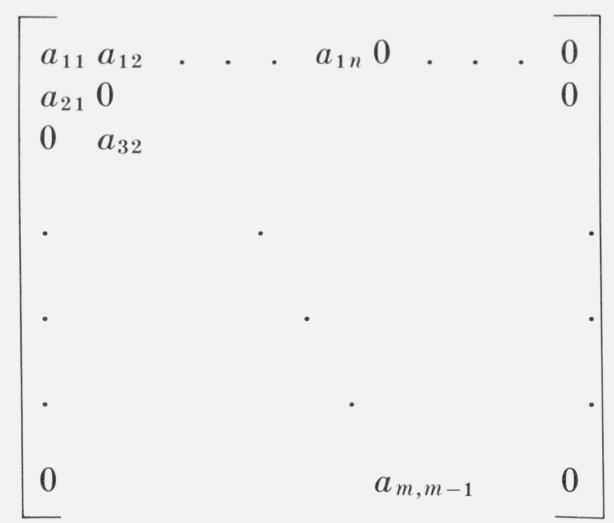

where $a_{11}, \ldots, a_{1 n}$ are age-specific fertility rates and $a_{21}, \ldots, a_{m, m-1}$ are survivership ratios, both of which are based on the time period $\Delta t$. In this paper we assume these rates remain constant.

AMS subject classification: $15 \mathrm{~A} 48 ; 92 \mathrm{A1}$

*Present address: Department of Mathematićs, University of Hawaii, Honolulu. 
$A$ matrix, $X$, with nonnegative entries is called reducible if there exist a permutation matrix $P$ such that

$$
P^{-1} X P=\left[\begin{array}{ll}
Y & 0 \\
W & Z
\end{array}\right]
$$

when $Y$ and $Z$ are square matrices. Matrices which are not reducible are called irreducible. The matrix (1) is reducible. However if we restrict ourselves to the first $n$ rows and columns we get the following irreducible matrix

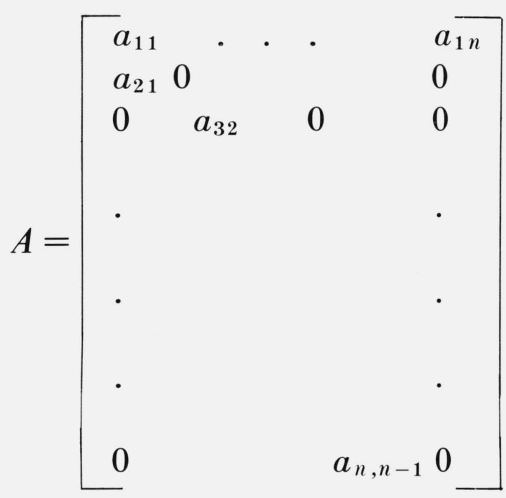

where $a_{1 n}$ is the last nonzero fertility rate. We also restrict $v(t)$ to the first $n$ components. This restricted model still contains all the essential information, see Parlett (1970).

The most important theorem for this population model is

Theorem 1: (Perron-Frobenius): Let A be an irreducible nonnegative (entry-wise) matrix. Then A has a simple positive eigenvalue $\mathrm{r}$ called the Perron root with corresponding eigenspace of dimension 1 and such that the absolute value of all other eigenvalues is less than or equal to $\mathrm{r}$. If A has exactly $\mathrm{h}$ roots with absolute value $\mathrm{r}$ then these roots are: $\mathrm{r}, \mathrm{re}^{\mathrm{i} 2 \pi / \mathrm{h}}, \mathrm{re}^{\mathrm{i} 4 \pi / \mathrm{h}}, \ldots, \mathrm{re}^{\mathrm{i}(\mathrm{h}-1) 2 \pi / \mathrm{h}}$. Furthermore, an eigenvector associated with $\mathrm{r}$ can be chosen to have positive components.

The proof of this theorem uses the Brouwer fixed point theorem. However, parts of this proof can be simplified for the matrix $A$ in (3). The characteristic polynomial of $A$ is

$$
f(\lambda)=\lambda^{n}-a_{11} \lambda^{n-1}-a_{12} a_{21} \lambda^{n-2}-a_{13} a_{32} a_{21} \lambda^{n-3} \ldots-a_{1 n} a_{n, n-1} a_{n-1, n-2} \ldots a_{21} .
$$

Since $f(0)<0$ and $f(x)>0$ for sufficiently large $x$, there is a positive root $r$, that is $f(r)=0$. To prove that $f(x)$ has no root with greater modulus than $r$ we need the following theorem due to Rouché see Nehari (1961), p. 138.

THEOREM 2:If $\mathrm{g}_{1}(\mathrm{z})$ and $\mathrm{g}_{2}(\mathrm{z})$ are polynomials over the complex numbers such that

$$
\left|\mathrm{g}_{1}(\mathrm{z})\right|>\left|\mathrm{g}_{2}(\mathrm{z})\right|
$$

everywhere on a circle $\mathrm{C}$, then the polynomial $\mathrm{g}_{1}(\mathrm{z})+\mathrm{g}_{2}(\mathrm{z})$ has the same number of roots, counting multiplicity, inside $\mathrm{C}$ as $\mathrm{g}_{1}(\mathrm{z})$.

It is clear from (4) that $f(R)>0$ for all $R>r$. Hence

$$
R^{n}>a_{11} R^{n-1}+a_{21} a_{12} R^{n-2}+a_{21} a_{32} a_{13} R^{n-3}+\ldots+a_{21} a_{32} \ldots a_{n, n-1} a_{1, n}
$$

for all $R>r$. Let $g_{1}(z)=z^{n}$ and $g_{2}(z)=f(z)-z^{n}$. Let $R>r$, then if $z$ is on the circle of radius $R$ about the origin, we get: 


$$
\begin{aligned}
\left|g_{2}(z)\right| & =\left|-a_{11} z^{n-1}-a_{21} a_{12} z^{n-2}-\ldots-a_{21} a_{32} \ldots a_{n, n-1} a_{1 n}\right| \\
& \leqslant a_{11} R^{n-1}+a_{21} a_{12} R^{n-2}+\ldots+a_{21} a_{32} \ldots a_{n, n-1} a_{1 n} \\
& <R^{n} \text { by (5). }
\end{aligned}
$$

It follows that all the roots of $f(z)$ lie within the circle $|z|=R$. Since $R$ can be made arbitrarily close to $r$, no eigenvalue can exceed $r$ in absolute value. We also point out that $r$ lies between the minimum and maximum row sums and also between the minimum and maximum column sums of (3). Since the row and column sums of (3) are of a very simple nature, in specific cases this may give fairly tight bounds on $r$. For example, in a human population the survivorship ratios $a_{i, i-1}$, $i=2, \ldots . . n$ may fall within a small range, and if total fertility, the sum $a_{11}+\ldots+a_{1 n}$ also falls in that range, then $r$ must fall in that range. For the proof of these and references to further inequalities see Gantmacher (1959).

In general even better bounds on $r$ can be attained from the $a_{i j}$ at the price of greater computation, see Marcus and Minc (1964). Perhaps the best of these applicable to an irreducible matrix of the form (3) is the following.

TheORem 3: Let $s$ be the minimum row sum and $S$ the maximum row sum of $A$. Let $\sigma$ be the sum of all entires of $A$ divided by $n$, and let $k$ be the smallest positive off diagonal entry of A. Set $\epsilon=(k / S)^{n-1}$. Then

$$
s+\epsilon(\sigma-s) \leqslant r \leqslant S-\epsilon(S-\sigma)
$$

We make one more definition which will be used later. An irreducible nonnegative matrix is called primitive if its Perron root is strictly larger than the absolute value of all other characteristic roots of $A$. A matrix which is not primitive is called imprimitive. A detailed discussion of primitivity is contained in Sykes (1969).

\section{Oscillations in Population}

It is well-known that under constant fertility and mortality the age specific population vector converges to a multiple of the eigenvector corresponding to the Perron root, provided that the population matrix is primitive. In this section we investigate what is happening to the population vector as it approaches its stable value. We will show that the population vector oscillates much like a damped pendulum, eventually coming to "rest" at the stable population vector.

Bernadelli (1941) discusses the importance and causes of these waves.

Let $\lambda_{1}=r, \lambda_{2}, \ldots, \lambda_{n}$ be the eigenvalues of the matrix $\dot{A}$ given in (3), numbered so that $\lambda_{1} \geqslant\left|\lambda_{2}\right| \geqslant \ldots \geqslant\left|\lambda_{n}\right|$. Let $r_{i}=\left|\lambda_{i}\right|$ and let $\theta_{i}$ be the angle $\lambda_{i}$ forms with the $x$ axis, $-\pi<\theta_{i} \leqslant \pi$. Assume for now that the eigenvalues of $A$ are distinct and that if $r_{i}=r_{j}$ then $i=j$ or $r_{i}$ is the complex conjugate of $r_{j}$. An account of what happens if these assumptions do not hold is given below. Let $w_{i}$ be an eigenvector of $A$ corresponding to the eigenvalue $\lambda_{i}$. Since $A w_{i}=\lambda_{i} w_{i}$ implies $\overline{\lambda_{i} w_{i}}=A \bar{w}_{i}=A \bar{w}_{i}$, where the bar denotes complex conjugate, we see that if $\lambda_{i}=\bar{\lambda}_{i+1}$ then we can choose $w_{i+1}=\bar{w}_{i}$. It is well-known from matrix theory that because $A$ has distinct eigenvalues any vector can be expressed as a linear combination of $w_{1}, \ldots, w_{n}$. So write

$$
v(0)=c_{1} w_{1}+c_{2} w_{2}+\ldots+c_{n} w_{n} .
$$

Taking the complex conjugate of both sides of (7) and using the fact that $\bar{v}(0)=v(0)$ we see that $c_{1}$ is real and that if $w_{i+1}=\bar{w}_{i}$ then $c_{i+1}=\bar{c}_{i}$. Applying $A^{t}$ to (7) gives

$$
v(t)=c_{1} r^{t} w_{1}+c_{2} \lambda_{2}^{t} w_{2}+\ldots+c_{n} \lambda_{n}^{t} w_{n} .
$$


If $\lambda_{2}$ is not real then $\lambda_{3}$ must be the complex conjugate of it. That means $r_{2}=r_{3}, \theta_{2}=-\theta_{3}$. Since $r_{i}<r_{2}$ for $i>3, v(t)$ can be approximated by

$$
\begin{aligned}
v(t) \cong c_{1} r^{t} w_{1} & +c_{2} \lambda_{2}^{t} w_{2}+c_{3} \lambda_{3}^{t} w_{3} \\
= & c_{1} r^{t} w_{1}+c_{2} r_{2}^{t}\left(\cos t \theta_{2}+i \sin t \theta_{2}\right) w_{2} \\
& +c_{3} r_{2}^{t}\left(\cos t \theta_{2}-i \sin t \theta_{2}\right) w_{3} \\
= & c_{1} r^{t} w_{1}+r_{2}^{t}\left[\cos \theta_{2} t\left(c_{2} w_{2}+c_{3} w_{3}\right)+\sin \theta_{2} t\left(c_{2} w_{2}-c_{3} w_{3}\right) i\right]
\end{aligned}
$$

for sufficiently large $t$.

Now $c_{1} r^{t} w_{1}$ is, of course, the stable part of the population and the other two terms, which are both real by the remarks above, give rise to the fluctuations from the stable population; that is, they give rise to waves. It is clear from (9) that the period of the oscillation is $2 \pi /\left|\theta_{2}\right|$. Also we see that the damping is determined by $\mathrm{r}_{2}$ : the size of the wave relative to the total population decrease at a rate of $r_{2} / r$ per time period.

If $r_{2}$ is real, then the $w_{3}$ term can be left out of the approximation in (9) and we have $\theta_{2}=\pi$ and thus the period will be two.

The case when $A$ is imprimitive is important for insect population. In this case $r_{2}=r$ which means the waves never die out. Suppose that $A$ has exactly $h$ eigenvalues whose absolute value is $r$. Then by Theorem 1 these eigenvalues are:

$$
\begin{gathered}
r, r e^{i \theta}, r e^{-i \theta}, r e^{i 2 \theta}, r e^{-i 2 \theta}, \ldots, r e^{i \frac{(h-1) \theta}{2}}, r e^{-i \frac{(h-1) \theta}{2}} \text { if } h \text { is odd } \\
r, r e^{i \theta}, r e^{-i \theta}, r e^{i 2 \theta}, r e^{-i 2 \theta}, \ldots, r e^{i \frac{h}{2} \theta} \text { if } h \text { is even }
\end{gathered}
$$

where $\theta=2 \pi / h$. The oscillations corresponding to these eigenvalues have periods of:

$$
\begin{array}{ll}
h, h / 2, h / 3, \ldots, 2 h / h-1 & \text { if } h \text { is odd } \\
h, h / 2, h / 3, \ldots, 2 h / h=2 & \text { if } h \text { is even. }
\end{array}
$$

From this we see that the waves have periods which have harmonic ratios. Note that the entire sum of the waves will repeat itself every $h$ time periods.

We now consider what happens if we relax some of our assumptions. If there are other eigenvalues, besides the complex conjugate of $\lambda_{2}$ which have the same absolute value as $\lambda_{2}$ and the eigenvalues of $A$ are still distinct, then the population waves would be the sum of the waves given by all the eigenvalues whose absolute value is $r_{2}$. In general the total fluctuation will be the sum of the waves generated by all the eigenvalues other than $r$. However the magnitude of oscillation of the eigenvalues $\lambda_{i}$ with $r_{i}<r_{2}$ will become small as compared to the magnitude of the oscillations of the eigenvalues with absolute value equal to $r_{2}$.

Now suppose $r>r_{2}=\left|\lambda_{2}\right|$ and that $\lambda_{2}=\lambda_{3}=\ldots=\lambda_{k}$ and that $r_{j}=\left|\lambda_{j}\right|<\left|\lambda_{2}\right|=r_{2}$ for $j>k$. Let $w_{1}, \ldots, w_{n}$ now be a basis which gives rise to the Jordan Normal Form, see Marcus and Minc, (1964). That is

$$
\begin{gathered}
A w_{1}=r w_{1}, \quad A w_{2}=\lambda_{2} w_{2}, \\
A w_{j}=w_{j-1}+\lambda_{j} w_{j} \quad j=3, \ldots, k .
\end{gathered}
$$


As before, we expand $v(0)$ in terms of $w_{1}, \ldots, w_{n}$. Applying $A^{t}$ to $v(0)$ and ignoring terms corresponding to eigenvalues whose absolute value is less than $r_{2}$, we get

$$
\begin{aligned}
& v(t) \cong c_{1} r^{t} w_{1}+ \\
& +\left(c_{2} \lambda_{2}^{t}+c_{3} t \lambda_{2}^{t-1}+\frac{c_{4} t(t-1)}{2 !} \lambda_{2}^{t-2}+\ldots+c_{k} \frac{t(t-1) \ldots(t-(k-3))}{(k-2) !} \lambda_{2}^{t-(k-2)}\right) w_{2} \\
& +\left(c_{3} \lambda_{2}^{t}+c_{4} t \lambda_{2}^{t-1}+\ldots+c_{k} \frac{t(t-1) \ldots(t-(t-4))}{(k-3) !} \lambda_{2}^{t-(k-3)}\right) w_{3}+\ldots+c_{k} \lambda_{2}^{t} w_{k} \\
& =c_{1} r^{t} w_{1}+\frac{t(t-1) \ldots(t-(k-3))}{(k-2) !} \lambda_{2}^{t-(k-2)}\left(\left[c_{2} \frac{(k-2) ! \lambda_{2}^{k-2}}{t(t-1) \ldots(t-(k-3))}+\ldots+c_{k}\right] w_{2}\right. \\
& \left.+\ldots+\frac{c_{k}^{(k-2)} ! \lambda_{2}^{k-2}}{t(t-1) \ldots(t-(k-3))} w_{k}\right) .
\end{aligned}
$$

Again for large $t$ this can be approximated by

$$
v(t) \cong c_{1} r^{t} w_{1}+c_{k} \frac{t(t-1) \ldots(t-(k-3))}{(k-2) !} \lambda_{2}^{t-(k-2)} w_{2} .
$$

We see from this equation that eventually the population vector does exhibit wave behavior. We can also see that the period of oscillation is the one which is associated with $\lambda_{2}$ and the relative damping from time period $t-1$ to $t$ is

$$
\frac{t}{(t-(k-2)) r}
$$

which approaches the usual value of $r_{2} / r$ for large $t$.

If there are several groups of eigenvalues all having absolute value $r_{2}$ then the overall wave will be a sum of terms as in (14), one such term for each group of eigenvalues. The behavior of these terms is more complicated but for large $t$ these terms become similar to the waves obtained in (9).

\section{Stability and the Relation Between the Perron Root and Other Demographic Variables}

A population is said to be stable over time if the percentage of the population in each age group is constant over time, that is the vector of population divided by the total population is constant over time.

It is well-known that a constant primitive transition matrix leads to stability over time.

As a converse we prove the following.

THEOREM 4: If a population exhibits stability over time then its transition matrix $\mathrm{A}(\mathrm{t})$ is constant if and only if the age specific birth rates are constant over time.

Proof: If the transition matrix $A(t)$ is constant over time, the birth rates must, of course, be constant. If the age specific birth rates, $a_{1 j}, j=1, \ldots, n$, are constant, then it suffices to note that in a stable population the survivorship ratios $a_{i, i-1}, i=2, \ldots, n$ are constant. This is the case since stability implies each age group is increasing at the same constant rate.

The theorem must be stated in this form since varying sets of birth rates could lead to essentially the same process if they deliver new cohorts increasing at the same rate as the population each time period. 
In a one sex population the net reproduction rate $(N R R)$ or expected number of births to a female allowing for the probabilities of mortality can be defined within the matrix model as the sum

$$
\begin{gathered}
a_{1,1}+a_{1,2} a_{2,1}+a_{1,3} a_{3,2} a_{2,1}+\ldots \\
+a_{1, n} a_{n, n-1} a_{n-1, n-2} \ldots a_{2,1} .
\end{gathered}
$$

The rate of natural increase $(R N I)$ of a stable population is merely the constant rate of increase it is experiencing in total numbers over time. Clearly $R N I=\lambda_{1}-1$ in a stable population, where $\lambda_{1}=r$ is the maximal positive eigenvalue. (A population is said to be stationary when $\lambda_{1}-1=0$.)

Since the NRR is somehow also a measure of how the population is increasing, intuition would expect a positive correlation between the $N R R$ and $R N I$ and thus $\lambda_{1}$. The following theorem states the greatest extent to which that intuition may be formalized.

THEOREM 5: (1) A stationary one-sex population is characterized by $\mathrm{RNI}=\left(\lambda_{1}-1\right)=(\mathrm{NRR}-1)=0$.

(2) In a stable one-sex population, (i) NRR $>1$ if and only if $\lambda_{1}>1$ and RNI $>0$ if and only if NRR $>\lambda_{1}$ and (ii) NRR $<1$ if and only if $\lambda_{1}<1$ and RNI $<0$ if and only if $\mathrm{NRR}<\lambda_{1}$.

Proof: The characteristic polynomial, det $(\lambda I-A)$ of our matrix form (3) was computed to be

$$
\begin{gathered}
f(\lambda)=\lambda^{n}-a_{1,1} \lambda^{n-1}-a_{1,2} a_{2,1} \lambda^{n-2}-a_{1,3} a_{3,2} a_{2,1} \lambda^{n-3}-\ldots \\
\ldots-a_{1, n} a_{n, n-1} a_{n-1, n-2} \ldots a_{21} \text { in (4). }
\end{gathered}
$$

It is important to note that the sum of all the subtracted coefficients is merely the NRR.

Now, in case (1), if $\lambda_{1}=1$ is a root of $f(\lambda)=0$, then $1=1-f(1)=N R R$ and conversely.

In case (2), $A / \lambda_{1}$ has maximum eigenvalue $\lambda_{1}^{\prime}=1$ by linearity. But this means that $1=a_{1,1} / \lambda_{1}+$ $a_{1,2} a_{2,1} / \lambda_{1}^{2}+a_{1,3} a_{3,2} a_{2,1} / \lambda_{1}^{3}+\ldots+a_{1, n} a_{n, n-1} a_{n-1, n-2} \ldots a_{2,1} / \lambda_{1}^{n}$ (from the characteristic polynomial of $A / \lambda_{1}$ ) which is equivalent to

$$
\lambda_{1}=a_{1,1}+a_{1,2} a_{2,1} / \lambda_{1}+a_{1,3} a_{3,2} a_{2,1} / \lambda_{1}^{2}+\ldots+a_{1, n} a_{n, n-1} a_{n-1, n-2} \ldots a_{2,1} / \lambda_{1}^{n-1} .
$$

Thus $\lambda_{1}>1$ if and only if

$$
a_{1,1}+a_{1,2} a_{2,1}+\ldots+a_{1, n} a_{n, n-1} a_{n-1, n-2} \ldots a_{2,1}>1
$$

if and only if $N R R>1$, and in this case $N R R$ must be greater than $\lambda_{1}$.

The proof is similar for $\lambda_{1}<1$ which completes the theorem.

Since the same $N R R$ could arise from many different sets of $a_{i, j}$ 's there is no more precise relation between the $N R R$ and $\lambda_{1}$.

\section{References}

Bernadelli, H., Population waves, Journal of Burma Research Society 3 1, 1-18 (1941).

Gantmacher, F. R., Theory of Matrices, Vol. 2 (Chelsea, New York, 1959).

Keyfitz, N., Introduction to the Mathematics of Population (Addison-Wesley, Reading, Mass., 1968).

Marcus, M., and Minc, H., A Survey of Matrix Theory and Matrix Inequalities (Allyn and Bacon, Boston, 1964).

Nehari, Z., Introduction to Complex Analysis (Allyn and Bacon, Boston, 1961).

Parlett, B., Ergodic Properties of Population I:The One Sex Model, Theoretical Population Biology, 1, 191-207 (1970).

Sykes, Z. M., On discrete stable population theory, Biometrics 25, 285-293 (1969).

(Paper 78B2-402) 